\title{
Steffek, J. und Holthaus, L. (Hrsg.) (2020). Prussians, Nazis and Peaceniks. Changing images of Germany in International Relations
}

\author{
Manchester: Manchester University Press. 256 S., ISBN: \\ 978-1-5261-3571-1, ca. € 110,-.
}

\section{Natalie Tröller}

Angenommen: 8. Februar 2021 / Online publiziert: 17. Februar 2021

(C) Der/die Autor(en) 2021

Was im eigenen, oftmals national verhafteten wissenschaftlichen Diskurs häufig außen vor bleibt, ist die Frage, wie die Dinge, deren man sich sehr sicher ist, von außenstehenden Beobachter*innen rezipiert werden. Kann man bisweilen von einer gewissen Betriebsblindheit sprechen, die der eigenen wissenschaftlichen Perspektive eigen sein mag, so ist die Öffnung dieser Perspektive durch Impulse von außen meist von großem Mehrwert, um eine Debatte anzureichern und zu diversifizieren. Diesem Versuch der Öffnung einer Debatte widmen sich Jens Steffek und Leonie Hotlhaus in ihrem Sammelband Prussians, Nazis and Peaceniks. Changing images of Germany in International Relations. ${ }^{1}$

Der Titel des Sammelbandes erzeugt zunächst in zweierlei Richtungen Erwartungen. Zum einen würden Leser*innen aufgrund des Verweises auf,,images of Germany in International Relations“ eine gründliche Globalschau von Perspektiven auf Deutschland erwarten. Diese Erwartung muss dahingehend eingegrenzt werden, dass sich der Sammelband hauptsächlich mit der US-amerikanischen Perspektive auf das un-, und das wiedervereinigte Deutschland sowie dessen historische Vorläufer auseinandersetzt. Zum anderen erweckt insbesondere der titelgebende Verweis auf die verschiedenen ,,images“ Deutschlands die Erwartung, es hier mit einem klassisch konstruktivistischen Werk zu tun zu haben, das sich anhand der Arbeit von Robert Jervis mit Rollenbildern, deren Konstruktion und Wandlung auseinandersetzt. Die

\footnotetext{
1 Der Band scheint das Ergebnis eines Workshops aus dem Jahr 2016 zu sein, der durch die Deutsche Forschungsgemeinschaft (DFG) über das Exzellenzcluster „Formation of Normative Orders“ gefördert wurde. Dieser Hinweis findet sich in einer Fußnote des Beitrages von Leonie Holthaus.
}

\section{N. Tröller $(\bowtie)$}

Lehrstuhl für Internationale Beziehungen und Außenpolitik, Universität Trier,

Universitätsring 15, 54296 Trier, Deutschland

E-Mail: troeller@uni-trier.de 
Herausgeber*innen möchten dazu anregen, eine breitere wissenschaftliche Debatte über das Rollenverständnis Deutschlands in den Internationalen Beziehungen zu verfolgen (s. xii), weisen allerdings darauf hin, dass ihr Verständnis von image nicht dem vielfach rezipierten Ansatz von Robert Jervis entspricht. Steffek und Holthaus begründen dies damit, dass Jervis Konzeption ,rather narrow“ (S. 5) sei und führen an: [It] ,seems to explore the phenomenon through its consequences“ (S. 5). Für Steffek und Holthaus ist ein image ,defined as a mental representation, impression or idea“ (S. 5). Entsprechend zählt für die Herausgeber*innen in dieser Konzeption die bildliche Darstellung deutscher Landschaften und Städte zu dessen image, wie auch Darstellungen des Dritten Reichs, der Militarisierung des Zweiten Weltkriegs oder der Schrecken des Holocausts. Sie unterstreichen mit Blick auf die folgenden Beiträge: ,[T]here never was just one image of Germany, but always several standing next to each other, sometimes compatible, sometimes contradictory“ (S. 6).

In den Beiträgen des vorliegenden Bandes wird das image Deutschlands dann aus verschiedenen Blickwinkeln illustriert. So widmet sich Andreas Osiander dem Machtbegriff als „deutsches Problem“ ausgehend vom Heiligen Römischen Reich bis in die Gegenwart. Gleichzeitig knüpft er dabei an die Diskussionen um die so genannten Sonderwegstheorien an, die auch in den Kapiteln von Leonie Holthaus und Annette Weinke anklingen. Holthaus beleuchtet die Konstruktion eines ,undemokratischen Deutschlands“ zu Beginn des zwanzigsten Jahrhunderts vonseiten liberaler Institutionalisten. Lucian M. Ashworth portraitiert die sich verändernde Betrachtung Deutschlands in angloamerikanischer Geopolitik. Paul Petzschmann beschäftigt sich in seinem Beitrag mit der Rezeption der Weimarer Republik durch die US-amerikanische Politikwissenschaft, Jens Steffek und Tobias Heinze portraitieren den US-Amerikaner Edwin M. Borchard (1884-1951), der laut ihnen als sehr früher Realist gelesen werden kann, in der akademischen Debatte aber nahezu vergessen ist. Annette Weinke betrachtet in ,the tale of the ,two Germanies “ [...] how twentieth-century Germany became something like a model case and a cautionary tale for international humanitarian law" (S. 123). Felix Rösch geht der Frage nach, welche Rolle Deutschland in der US-amerikanischen Wahrnehmung Internationaler Beziehungen nach dem zweiten Weltkrieg spielte. Brian C. Etheridge betrachtet „Deutschtum and Americanism“ als Pfeiler jeweiliger Identitäten im Kalten Krieg. Siegfried Schieder nimmt Bezug auf die Betrachtung der ,Zivilmacht Deutschland" aus US-amerikanischer Perspektive und illustriert unter Rückgriff auf das Konzept der Zivilmacht von Hanns W. Maull die „Dissonanz zwischen der Selbstwahrnehmung Deutschlands und der Wahrnehmung anderer, die zunehmend zu Unstimmigkeiten führt" (S. 186). Er spricht beispielhaft Ereignisse aus der jüngeren Vergangenheit an, wie die Enthaltung Deutschlands bei der Abstimmung zur Sicherheitsratsresolution 1973, Deutschlands Rolle in der Syrien-Politik oder dessen Reaktion auf die Flüchtlingsbewegungen 2015 - Fragestellungen, die sich für eine weitere kritische Diskussion auch in zusätzlichen Beiträgen angeboten hätten. Der Sammelband schließt mit einem Fazit von Richard Ned Lebow, in dem sich Lebow empirisch mit den Einfluss deutscher und deutschsprachiger Wissenschaftler*innen auf die Entwicklung und Fortentwicklung des wissenschaftlichen Diskurses in den Internationalen Beziehungen auseinandersetzt. 
Insgesamt verfolgt der Band einen interessanten Ansatz, einen Perspektivenwechsel einzunehmen und verschiedene Außenperspektiven auf Deutschland in den Internationalen Beziehungen zu Wort kommen zu lassen. Die in einigen Beiträgen stark historisch orientierte Perspektive kann als interessante Ergänzung aufgefasst werden, sich mit den Ursprüngen vorherrschender images in den Internationalen Beziehungen auseinander zu setzen. Zusätzlich wäre hier die Öffnung gegenüber weiteren Perspektiven und aktueller Fragestellungen insbesondere abseits klassischer transatlantischer Ansätze spannend gewesen, um das hier portraitierte image Deutschlands in den Internationalen Beziehungen weiter zu diversifizieren.

Funding Open Access funding enabled and organized by Projekt DEAL.

Open Access Dieser Artikel wird unter der Creative Commons Namensnennung 4.0 International Lizenz veröffentlicht, welche die Nutzung, Vervielfältigung, Bearbeitung, Verbreitung und Wiedergabe in jeglichem Medium und Format erlaubt, sofern Sie den/die ursprünglichen Autor(en) und die Quelle ordnungsgemäß nennen, einen Link zur Creative Commons Lizenz beifügen und angeben, ob Änderungen vorgenommen wurden.

Die in diesem Artikel enthaltenen Bilder und sonstiges Drittmaterial unterliegen ebenfalls der genannten Creative Commons Lizenz, sofern sich aus der Abbildungslegende nichts anderes ergibt. Sofern das betreffende Material nicht unter der genannten Creative Commons Lizenz steht und die betreffende Handlung nicht nach gesetzlichen Vorschriften erlaubt ist, ist für die oben aufgeführten Weiterverwendungen des Materials die Einwilligung des jeweiligen Rechteinhabers einzuholen.

Weitere Details zur Lizenz entnehmen Sie bitte der Lizenzinformation auf http://creativecommons.org/ licenses/by/4.0/deed.de. 